\title{
Systemic immune-inflammation index and prognosis of advanced non-small cell lung cancer
}

\author{
Marco Alifano ${ }^{1,2}$ \\ ${ }^{1}$ Department of Thoracic Surgery, Cochin Hospital, AP-HP Centre - University of Paris, Paris, France; ${ }^{2}$ Team Cancer, Immune Control and Escape, \\ INSERM U1138, Cordeliers Research Center, Paris, France \\ Correspondence to: Prof. Marco Alifano, MD, PhD. Department of Thoracic Surgery, Cochin Hospital, AP-HP Centre - University of Paris, Paris, \\ France; Team Cancer, Immune Control and Escape, INSERM U1138, Cordeliers Research Center, 27 rue du Faubourg Saint Jacques, 75014 Paris, \\ France. Email: marco.alifano@aphp.fr. \\ Provenance and Peer Review: This article was commissioned and reviewed by the Academic Editor Dr. Shuangjiang Li (Department of Thoracic \\ Surgery and West China Medical Center, West China Hospital, Sichuan University, Chengdu, China). \\ Comment on: Berardi R, Santoni M, Rinaldi S, et al. Pre-treatment systemic immune-inflammation represents a prognostic factor in patients with \\ advanced non-small cell lung cancer. Ann Transl Med 2019;7:572.
}

Submitted Feb 24, 2020. Accepted for publication Mar 26, 2020.

doi: 10.21037/atm.2020.03.174

View this article at: http://dx.doi.org/10.21037/atm.2020.03.174

Management of patients with non-small cell lung cancer (NSCLC) is based on histologic diagnosis and staging. Although stage of disease at diagnosis remains the mainstay of treatment, its exact prognostic significance is matter of debate (1). Patients' outcome is not homogeneous within the same stage and after the same treatments; this led evaluating other tumor-related factors (vascular or lymphatic emboli, tumor grade) possibly influencing survival $(2,3)$. Similarly, mutations of driver oncogenes (which represent the basis for currently employed targeted therapies) have been assessed as a prognostic indicators, with different mutations associated to more favorable or, conversely, unfavorable outcome (4). Although less extensively studied, deregulation of cancer cell metabolism, favoring replicative immortality, invasion and metastasis has also been suggested as a potential determinant of cancer progression (5).

Thus, extensive literature exists on prognostic impact of tumor-related factors, whereas host-related factors have been much less deeply evaluated. Systemic inflammation has been shown to be present in approximately half of operable (stage I-III) patients with NSCLC and measure of plasma $\mathrm{C}$ reactive protein (CRP) levels is considered an excellent marker of this condition (6); although this finding agrees with the well-established relationship between CRP levels and burden of primary cancer [for example, the number of patients with increased CRP levels is relatively low in stage IA $(30 \%)$ but increases dramatically in stage IB (60-70\%) NSCLC], the independent prognostic impact of CRP levels on one side and of $\mathrm{pT}$ parameter on the other, is proven: this would suggests that tumor bulk and systemic inflammation are correlated but independent predictors (6).

A recent cohort study performed by Berardi et al., entitled "Pre-treatment systemic immune-inflammation represents a prognostic factor in patients with advanced non-small cell lung cancer", published in the Annals of Translational Medicine (7), enrolled 311 patients with advanced NSCLC undergoing first-line chemo- or targeted therapy and evaluated the prognostic value of Systemic Immune-Inflammation Index (SII) for both overall survival and progression-free survival. SII is a composite index taking into account platelet, neutrophil and lymphocyte counts [SII $=$ (platelet $\times$ neutrophils)/lymphocyte count $]$. The main findings of Berardi et al. included: (I) both overall survival and progression-free survival were significantly lower in the higher SII group than in the lower SII group; (II) higher dichotomized SII was one of the leading prognostic factors of survival (overall and progressionfree) of advanced NSCLC. These results are extremely interesting and confirm that a better understanding of putative inflammatory indicators could play a vital role in assisting physicians to identify which patients are considered 
at a higher probability of unfavorable prognosis, justifying an appropriate management plan in advance. Thus, Berardi et al. (7) brought another significant stone for building of knowledge that host-specific factors are as important as tumor factors in determining the outcome of NSCLC. However, how systemic inflammation worsens the prognosis of lung cancer patients remains matter of speculation.

It has been suggested that, in resectable lung cancer, systemic inflammation could help cancer cells to maintain a microenvironment favoring survival of remnant cells after a resection considered complete $(1,6)$. Pro-inflammatory cytokines and associated growth factors participate in carcinogenesis and tumor development tanks to their actions on cancer cell growth, survival, proliferation and migration $(1,6)$. Of note, high CRP levels are associated with the existence of neoplastic vascular emboli, and microscopic invasion of hematic and lymphatic vessels is a key step of tumor spread (6). The prognostic significance of high CRP could be explained by the relationship existing between systemic inflammation and nutritional status (8). Chronic inflammation induces catabolic processes and reduced caloric intake due to the malignancy, symptoms and, possibly, treatments, may be at the origin of fat and muscle loss (9). We found that lower BMI and sarcopenia were independent negative prognostic factors in lung cancer requiring pneumonectomy, together with higher CRP levels (10). The negative impact of poor nutritional status and sarcopenia concerned both short-term (post-operative) and long-term period (10). We confirmed these findings in a larger patient cohort of NSCLC undergoing all kind of resection and showed that not-only pre-surgery BMI but also pre-disease BMI affected long-term survival (11). In patients undergoing pneumonectomy, BMI and total psoas area (a measure of sarcopenia) were strongly and directly correlated; BMI was inversely related to CRP levels, whereas sarcopenia was associated with high CRP levels, underlining the important interplay between systemic inflammation, nutritional status and muscle waste (10).

Systemic inflammation could also exert a deleterious effect on lung cancer through interaction with tumoral immune microenvironment (8), which is a major prognostic determinant in resected primary lung cancer: high levels of mature dendritic cells (mDC) and of CD8+ lymphocytes in the operative specimens are robust independent positive prognostic factors, reflecting the function of a wellorganized anti-tumor immune microenvironment $(8,12)$. The presence of B lymphocytes contributes to function of these tertiary lymphoid structures and is associated to a better outcome as well (13). Of note, induction chemotherapy in stage IIIA disease does not alter the tumor immune contexture, nor its prognostic significance, supporting the concept of potential beneficial synergistic effect of immunotherapy and chemotherapy (14).

It has been observed that the number of $\mathrm{mDC}$ and CD8+ lymphocytes in operative specimens is directly correlated with albumin and pre-albumin levels (indicators of nutritional status) and inversely correlated with pre-operative CRP levels (indicator of systemic inflammation) (8). Furthermore, correlations exist also between the number of CD8+ $\mathrm{T}$ cells and $\mathrm{mDC}$ in the resection specimen and the existence of several associated conditions and clinical features (such as previous stroke, chronic bronchitis, usual body weight), suggesting that poor nutritional status and/or chronic systemic inflammation could impact the intra-tumor immune contexture and the patient prognosis, possibly through a complex interplay (8). In the specific sub-group of resected lung cancer with COPD, a condition also associated with systemic inflammation, we observed that Global Initiative for Chronic Lung Disease (GOLD) stage of COPD was directly correlated with the coexpression of PD-1/TIM3 (T-cell immunoglobulin and mucin domain-containing molecule-3) by CD8 T cells, suggesting the exhaustion of these cells, and, in agreement, a loss of favorable prognosis of CD8 $\mathrm{T}$ cell infiltration in patients with COPD (15).

Overall, large scale analysis of immunes cells shows clearly that even beyond complexity of immune environment, CD $8+\mathrm{T}$ cells inside the immune microenvironment are generally protective, meanwhile CD4+ regulatory $\mathrm{T}$ cells (Tregs) and neutrophils would exert a pro-tumoral action (16). Thus, the tumour stroma, which hosts the tumor immune environment, should be considered as a complex medium, composed of both "good" and "bad" immune cells, whose mutual interaction have not been clearly analysed yet although it may explain immunotherapy failure in a large percentage of patients in most cancers, including NSCLC. Although neutrophilia has been considered a negative prognostic factor of NSCLC for several years, other parameters, taking into account neutrophil count have been more extensively assessed in the last few years, including the neutrophil/lymphocyte ratio, and, even more recently, the SII, which takes into account also platelets.

Platelets are involved in occurrence of metastasis: circulating tumor cells adhere to the activated platelets forming tumor microthrombi. Thanks to adhesion 
molecules, these microthrombi can adhere to endothelial cells in microvessels: this is the first step in the genesis of hematogenous metastases. The molecules involved in adhesion to platelets and the vascular endothelium are the surface glycosaminoglycans of tumor cells which are modified and contain a tetrasaccharide of the sialyl Lewis $\mathrm{X}$ or sialyl Lewis A type, whose presence is associated with a poor tumor prognosis $(17,18)$. Platelet and endothelial receptor for these modified glycosaminoglycans is $\mathrm{P}$-selectin, whose depletion is associated with a reduction in metastases in animal models $(17,18)$. It has been suggested that higher concentration of platelets could more easily lead to the formation of venous thrombi. It has also been pointed out that platelets would act as "cloaks" for circulating tumor cells by shielding them from the attack of natural killer (NK) cells. Finally, platelets secrete a variety of growth factors and angiogenesis-regulating proteins promoting tumor growth and metastasis. It should be underlined that interactions between platelets and cancer cells are to be considered as specular, that means that cancer cells might first stimulate activity of platelet (together with and increased production), and then platelets might enhance tumor growth, invasion, and metastasis $(19,20)$.

Neutrophils are main actors of acute inflammation and infection, and play a major role in innate immunity; their role in cancer immunity is less known as compared to CD8+ or mDC. Analyses of tumor specimen showed that neutrophils represent a large proportion of immune cells, and a negative correlation between counts of neutrophils and T cells (both CD4 and CD8) has been reported, which is in agreement with their putative immunosuppressive functions in cancers (21).

Among neutrophils, different subtypes exists and, differently from healthy volunteers, who have normal density neutrophils (NDNs), in cancer patients a subpopulation of neutrophils with strong immunosuppressive functions can be found in both blood and tissue, namely the low density neutrophils (LDNs), which have been suggested to be PMN-MDSCs (polymorphonuclear myeloid-derived suppressor cells) (22). These PMN-MDSCs may express PD-L1 and could exert their immunosuppressive function in cancer through PDL1 axis. Inhibition of STAT3 may decrease the population of MDSCs and increase the number of local tumorinfiltrating lymphocytes, representing a promising new way to enhance efficacy of immunotherapies (22).

Tumor-infiltrating neutrophils are derived from circulating neutrophils and accumulating evidence (including the work of Berardi $e t a l$.) on deleterious effect of high concentration of circulating neutrophils could be regarded in the light of their impact on constitution and function of tumoral immune environment: thus translation from knowledge of function of tumoral neutrophils to circulating ones warrants urgent, specific studies in large series of lung cancer patients with both localized and disseminated disease.

\section{Acknowledgments}

Funding: None.

\section{Footnote}

Conflicts of Interest: The author has completed the ICMJE uniform disclosure form (available at http://dx.doi. org/10.21037/atm.2020.03.174). The author has no conflicts of interest to declare.

Ethical Statement: The author is accountable for all aspects of the work in ensuring that questions related to the accuracy or integrity of any part of the work are appropriately investigated and resolved.

Open Access Statement: This is an Open Access article distributed in accordance with the Creative Commons Attribution-NonCommercial-NoDerivs 4.0 International License (CC BY-NC-ND 4.0), which permits the noncommercial replication and distribution of the article with the strict proviso that no changes or edits are made and the original work is properly cited (including links to both the formal publication through the relevant DOI and the license). See: https://creativecommons.org/licenses/by-nc-nd/4.0/.

\section{References}

1. Bobbio A, Alifano M. Immune therapy of non-small cell lung cancer. The future. Pharmacol Res 2015;99:217-22.

2. Strano S, Lupo A, Lococo F, et al. Prognostic significance of vascular and lymphatic emboli in resected pulmonary adenocarcinoma. Ann Thorac Surg 2013;95:1204-10.

3. Mansuet-Lupo A, Bobbio A, Blons H, et al. The new histologic classification of lung primary adenocarcinoma subtypes is a reliable prognostic marker and identifies tumors with different mutation status: the experience of a French cohort. Chest 2014;146:633-43.

4. Mansuet-Lupo A, Alifano M, Pécuchet N, et al. 
Intratumoral Immune Cell Densities Are Associated with Lung Adenocarcinoma Gene Alterations. Am J Respir Crit Care Med 2016;194:1403-12.

5. Icard P, Fournel L, Wu Z, et al. Interconnection between Metabolism and Cell Cycle in Cancer. Trends Biochem Sci. 2019;44:490-501.

6. Alifano M, Falcoz PE, Seegers V, et al. Preresection serum C-reactive protein measurement and survival among patients with resectable non-small cell lung cancer. J Thorac Cardiovasc Surg 2011;142:1161-7.

7. Berardi R, Santoni M, Rinaldi S, et al. Pre-treatment systemic immune-inflammation represents a prognostic factor in patients with advanced non-small cell lung cancer. Ann Transl Med 2019;7:572.

8. Alifano M, Mansuet-Lupo A, Lococo F, et al. Systemic inflammation, nutritional status and tumor immune microenvironment determine outcome of resected nonsmall cell lung cancer. PLoS One 2014;9:e106914.

9. Icard P, Iannelli A, Lincet H, et al. Sarcopenia in resected non-small cell lung cancer: let's move to patient-directed strategies. J Thorac Dis 2018;10:S3138-42.

10. Hervochon R, Bobbio A, Guinet C, et al. Body Mass Index and Total Psoas Area Affect Outcomes in Patients Undergoing Pneumonectomy for Cancer. Ann Thorac Surg 2017;103:287-95.

11. Icard P, Schussler O, Loi M, et al. Pre-Disease and PreSurgery BMI, Weight Loss and Sarcopenia Impact Survival of Resected Lung Cancer Independently of Tumor Stage. Cancers (Basel) 2020. doi: 10.3390/cancers12020266.

12. Goc J, Germain C, Vo-Bourgais TK, et al. Dendritic cells in tumor-associated tertiary lymphoid structures signal a Th1 cytotoxic immune contexture and license the positive prognostic value of infiltrating CD8+ T cells. Cancer Res 2014;74:705-15.

13. Germain C, Gnjatic S, Tamzalit F, et al. Presence of B cells in tertiary lymphoid structures is associated with a

Cite this article as: Alifano M. Systemic immuneinflammation index and prognosis of advanced non-small cell lung cancer. Ann Transl Med 2020;8(11):667. doi: 10.21037/ atm.2020.03.174 protective immunity in patients with lung cancer. Am J Respir Crit Care Med. 2014;189:832-44.

14. Remark R, Lupo A, Alifano M, et al. Immune contexture and histological response after neoadjuvant chemotherapy predict clinical outcome of lung cancer patients.

Oncoimmunology 2016;5:e1255394.

15. Biton J, Ouakrim H, Dechartres A, et al. Impaired Tumor-Infiltrating T Cells in Patients with Chronic Obstructive Pulmonary Disease Impact Lung Cancer Response to PD-1 Blockade. Am J Respir Crit Care Med 2018;198:928-40.

16. Gentles AJ, Newman AM, Liu CL, et al. The prognostic landscape of genes and infiltrating immune cells across human cancers. Nat Med 2015;21:938-45.

17. Borsig L, Wong R, Feramisco J, et al. Heparin and cancer revisited: mechanistic connections involving platelets, P-selectin, carcinoma mucins, and tumor metastasis. Proc Natl Acad Sci U S A 2001;98:3352-7.

18. Alifano M, Benedetti G, Trisolini R. Can low-molecularweight heparin improve the outcome of patients with operable non-small cell lung cancer? An urgent call for research. Chest 2004;126:601-7.

19. Baranyai Z, Krzystanek M, Jósa V, et al. The comparison of thrombocytosis and platelet-lymphocyte ratio as potential prognostic markers in colorectal cancer. Thromb Haemost 2014;111:483-90.

20. Rao XD, Zhang H, Xu ZS, et al. Poor prognostic role of the pretreatment platelet counts in colorectal cancer. A meta-analysis. Medicine (Baltimore) 2018;97:e10831.

21. Kargl J, Busch SE, Yang GH, et al. Neutrophils dominate the immune cell composition in non-small cell lung cancer. Nat Commun 2017;8:14381.

22. Gabrilovich DI, Nagaraj S. Myeloid-derived suppressor cells as regulators of the immune system. Nat Rev Immunol 2009;9:162-74. 